\title{
Linearization Approach of Combined Digital Predistortion with Crest Factor Reduction
}

\author{
Zhinian Luo, Zhenpeng Huang ${ }^{+}$ \\ School of Hunan University, Changsha 410082, China
}

\begin{abstract}
In recent years, digital predistortion (DPD) has been wildly used in the nonlinear compensation of power amplifier. However, saturation distortion which caused by high peak-to-average ratios (PAPR) of the input signal cannot be compensated by DPD perfectly. Therefore, power amplifier should work in certain back-off and results in low efficiency. In this paper, a new method of combined adaptive DPD and crest factor reduction (CFR) is proposed to reduce PAPR, which uses selective mapping (SLM) and companding transform (CT). Besides, an adaptive DPD utilized the LS-LMS algorithm is proposed, which shows stable and fast convergence in the proposed system. Simulation results show that the proposed method can achieve a desirable linearization performance and decrease the predistorted signal's PAPR by $7 \mathrm{~dB}$.
\end{abstract}

Keywords: digital predistortion, power amplifier, selective mapping, adaptive, companding transform.

\section{Introduction}

Power amplifier (PA), the essential key components in the wideband wireless communication system, is often working in the saturation region to improve the efficiency. However, the PA work in the saturation region will produce nonlinear distortion. Furthermore, the transmitted signal could be effect by the nonlinearities, such as the inter-symbol interferences and spectral regrowth. Therefore, it is necessary to use PA linearization techniques to compensate the nonlinearity of PA. Currently, the most common techniques used in PA linearization include power back-off (PBO), feed-forward predistortion (FFP), linear amplification with nonlinear components (LINC) and digital predistortion. By now, DPD has become the predominant technique for PA linearization because of its strong stability, high effectiveness and easy implementation [1].

With the rapidly development of digital communication techniques, modern $3 \mathrm{G}$ and $4 \mathrm{G}$ communication standards adopt signals with high spectral efficiency to support the increased request of elevated data rates. Non-constant envelope modulation schemes orthogonal frequency division multiplexing (OFDM) is widely deployed. It offers a considerable high spectral efficiency, multipath delay spread tolerance and power efficiency and very strong immunity to the frequency selective fading channels. However, one majority drawbacks of OFDM is the high peak-to-average power ratio (PAPR) [2] which seriously limits the power efficiency and causes a nonlinear distortion. The nonlinear and memory effects are particularly serious when the OFDM signals go through the PA. The effects of memory can be resolved by inserting a memory model in preditorter. However, the saturation distortion caused by PAPR cannot be compensated well with DPD [3]. Byung Moo Lee and Rui J.P.de Figueiredo proposed an optimum level of power back off solution for this problem in the [4], which gives a criterion to the need of power back-off. But, this power back-off is not desirable because it reduces the power efficiency. Farabegoli proposed a combining CFR and adaptive DPD in the [5], which demonstrated the strength of combination totally. But it does not propose specific solutions. At present, many schemes of combined single clipping method of DPD have been proposed, such as [3], [6].

\footnotetext{
+ Corresponding author. Tel: +8615575861842

E-mail address: 610207921@qq.com.
} 
These schemes have good performance in the PAPR reduction. But it can easily introduce clipping noise, which affects the performance of the whole system.

In this paper, a new approach of combining CFR and adaptive DPD is proposed. First, we use the SLM to reduce the peak signal of PAPR. Then, CT method is used to reduce the PAPR further.

Finally, with computer simulation, the results show that the proposed method has better performance in reducing PAPR than the single clipping method. The paper is organized as follow: The first part introduces two methods of PAPR reduction: SLM and CT. The second part introduces adaptive DPD and the proposed new structure of combined SLM+CT and adaptive DPD. The simulation results analysis in the third section. Conclusions are given in the final.

\section{CFR Overview}

The techniques of signal PAPR reduction are attributed to three categories: the signal predistortion techniques, such as direct clipping method [7] and companding transform (CT).The probability techniques, such as selective mapping (SLM) [8] and PTS [9]. The last one is coding techniques. In this paper, the methods of CT and SLM are described mainly.

\subsection{Companding Transform}

CT is a signal predistortion method based on numerical transform and reduced the PAPR with altering signal amplitude. The conventional CT amplifies small signal and keeps the lager signal unchanged, which can enhance the average signal power. However, the PA will working in the nonlinear region with the larger average signal. Therefore, a new approach, C-transform method, has been proposed. It is not only can amplify the small signal but also can compress the lager signal. Moreover, it can maintain the average power to improve the systems resistance interference and reduce the PAPR. The CT procedure can be expressed as Equation (1)

$$
X^{\prime}(t)=C[X(t)]
$$

where $C[$.$] is the CT function. Currently, we always use CT methods included the A-CT and the \mu-\mathrm{CT}$. The paper focuses on the $\mu$-CT to reduce PAPR. The CT functions is expressed as [7]

$$
s^{\prime}(n)=\frac{A \operatorname{sgn}\left[s ( n ) \operatorname { l n } \left[1+\mu\left|\frac{s(n)}{A}\right|\right.\right.}{\ln (1+\mu)}
$$

where $A$ is the amplitude normalizations factor, $0 \leq\left|\frac{s(n)}{A}\right| \leq 1$, and $s(n)$ is discrete time-domain OFDM signal.

\subsection{Selective Mapping}

The core idea of SLM method is first using different sequences, in which every sequence has the same phase factor, to interference the frequency domain OFDM signal. Then, the symbol with smallest PAPR is selected to transmit. Block diagram of SLM [Fig. 1] and specific process is expressed as follows [8]:

The input data block $X=[\mathrm{x}[0], \mathrm{x}[1], \ldots, \mathrm{x}[\mathrm{N}-1]$ multiplied with a phase sequences $P^{u}=\left[p_{0}^{u}, p_{1}^{u}, \ldots, p_{N-1}^{u}\right]$ so that a modified block $X^{u}=\left[\mathrm{x}[1]^{u}, \mathrm{x}[2]^{u}, \ldots, \mathrm{x}[\mathrm{N}-1]^{u}\right]^{T}$ obtained, where $P_{v}^{u}=e^{j \phi_{v}^{u}}, \phi_{v}^{u} \in[0,2 \pi), v=0,1, \ldots, N-1, u=1,2, \ldots, U$. After an IFFT transform to $\left\{X^{u}[\mathrm{v}]\right\}$, we can obtain a new sequence $x^{u}=\left[\mathrm{x}[0]^{u}, \mathrm{x}[1]^{u}, \ldots, \mathrm{x}[\mathrm{N}-1]^{u}\right]^{T}$. Finally, a sequence that has a minimum PAPR is selected to transmit. In order to recover the original block data from the receiver, we should use $U$ as the transmitting side information. 


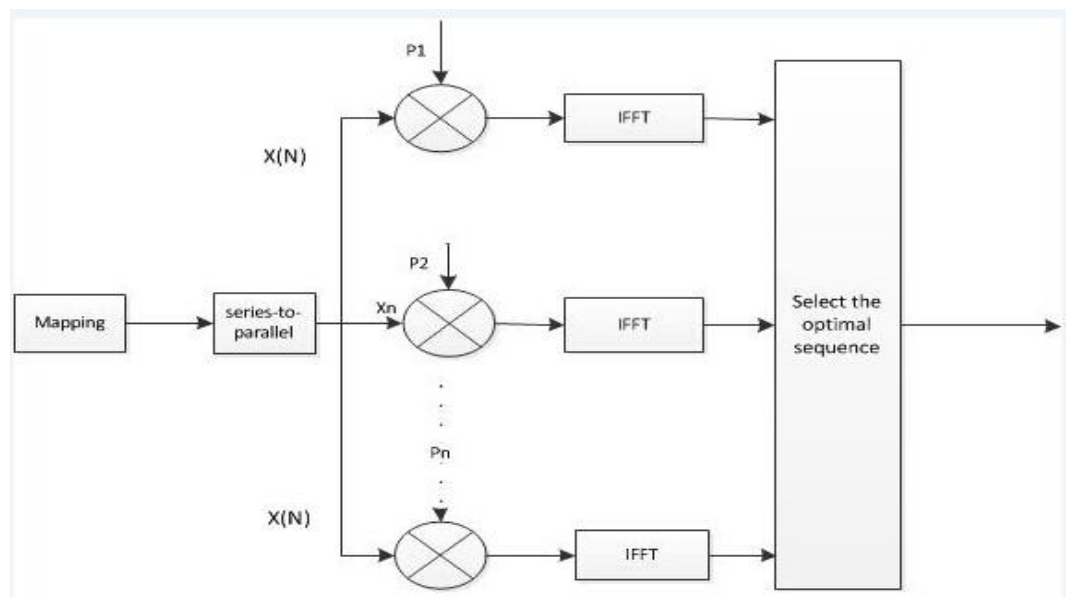

Fig. 1: SLM schematic diagram.

\section{The Proposed DPD}

\subsection{HPA Model}

Judging from the PA has the memory effect or not, PA model can be divided into two categories: memory-less PA behavioural models and memory PA behavioural models. The memory-less PA behavioural models mainly include Saleh model and Rapp model. They are extensively used in the narrowband signal without considering the memory effects. The memory PA behavioural models contain Volterra model, wiener model, Hammerstein model, memory polynomial (MP) model and neural network models, which are used in the nonlinear memory PA systems. In this paper, we use the memory model due to the wide broadband of the OFDM signal. The MP model has better performance than Weiner model and Hammerstein model in the complexity computation and the model's accuracy [10]. The MP function is expressed as

$$
y(n)=\sum_{k=0}^{K-1} \sum_{q=0}^{Q-1} c_{k q} \mathrm{z}(n-q)|\mathrm{z}(n-q)|^{k}
$$

where $z(n), y(n)$ is the input digital signal and output digital signal, respectively. And the polynomial is $\mathrm{K}$ and the memory depth is $\mathrm{Q}$.

\subsection{Conventional DPD}

PA's nonlinear characteristics can be described as amplitude modulation to amplitude modulation conversion (AM/AM) and amplitude modulation to phase modulation conversion (AM/PM).DPD is in control of producing inverse AM/AM and AM/PM curves to cancel the AM/AM and AM/PM nonlinear characteristics of PA. Namely, the predistortion curves and the distortion curves are complementary to achieve linear amplification.

A typical digital predistortion system is show as Fig. 2. The digital baseband signals predistored by the DPD block before being converted to the analogy domain and modulated to the RF frequency and finally fed into the PA.

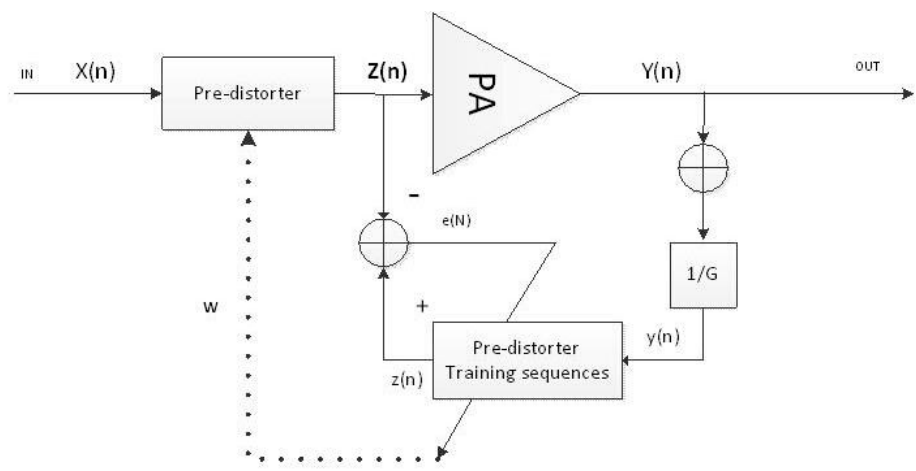

Fig. 2: Diagram of adaptive digital predistortion indirect learning structure. 


\subsection{Adaptive Estimation Algorithms}

The adaptive algorithm module plays an important role in the adaptive DPD. Adaptive identification module [Fig. 3] is separated out from the system block diagram of the adaptive DPD system.

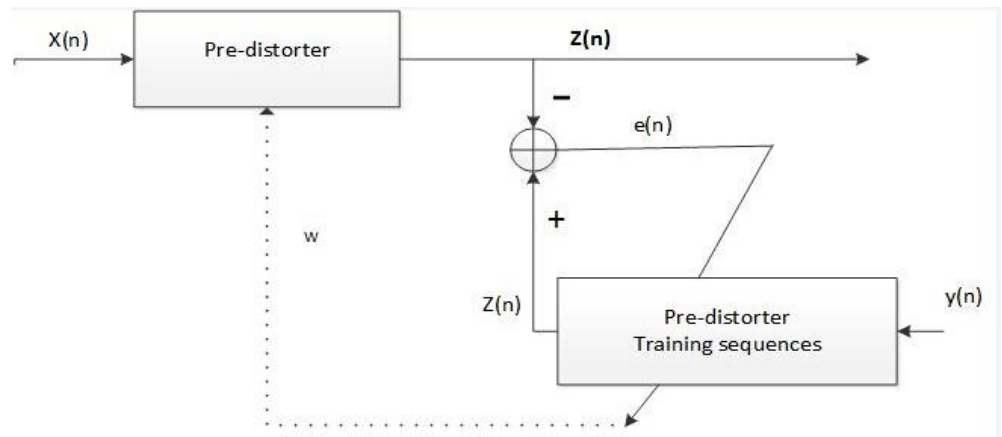

Fig. 3: Adaptive identification system block diagram.

Here, $\mathrm{x}(\mathrm{n})$ is the input signal and $\mathrm{y}(\mathrm{n})$ is the output signal. $z(n)$ is the actual output signal and $z \hat{(n)}$ is the expected output signal. The preditorter training sequences block adopts the MP model. The T-MP is expressed as

$$
z \hat{(n)}=\sum_{k=0}^{K-1} \sum_{q=0}^{Q-1} c_{k q} \mathrm{y}(n-q)|\mathrm{y}(n-q)|^{k}
$$

where $e(n)$ is the signal error, the equation is expressed as,

$$
e(n)=z(n)-z \hat{(n)}
$$

Adaptive estimation algorithm is used to minimize the target function of signal error e(n) . Generally, the adaptive estimation algorithms primarily include least mean square (LMS) and the least square (LS).

\subsubsection{LS}

In the practical communication systems, the length of signal is always limited. So we can substitute the mean square of error in the (10) for the error sum of squares. The LS target function can be wrote as

$$
\varepsilon(\mathrm{n})=\sum_{\mathrm{n}=0}^{N-1}\left[z(\mathrm{n})-\mathrm{W}_{N}(n) \mathrm{Y}_{N}(n)\right]^{2}
$$

where $N$ is fewer snapshots.

To make the target function's gradient reach zero, we will get the optimal weighted vector $\vec{w}$ of LS. From the (4), setting $u(n)$ as follow

$$
u(n)=c_{k q} \mathrm{y}(n-q)|\mathrm{y}(n-q)|^{k}
$$

when the convergence criteria in (4) is satisfied,

$$
\vec{Z}=\vec{U} \cdot \vec{w}
$$

where $\vec{Z}=[\mathrm{z}(0), \cdots \mathrm{z}(N-1)]^{T} ; \vec{U}=\left[u_{1}, \cdots, u_{k}\right], \vec{u}_{k}=[u(0), \cdots, u(N-1)]^{T} ; \quad \vec{w}=\left[w_{1}, \cdots w_{k}\right]^{T}$.

The solution of LS in equation (8) is expressed as

$$
\vec{w}=\left(\mathrm{U}^{H} U\right)^{-1} U^{H} Z
$$

In which $U^{H}$ indicates the conjugate transpose of a complex matrix $U$. When the indirect learning DPD algorithm is applied, by swapping the input and output of the PA in (9), the DPD parameters can be obtained [11]. 


\subsubsection{LMS}

LMS algorithm [12], based on the minimum mean square error rule with steepest descent, is widely used in adaptive algorithm due to its low complexity, fast convergence and small error.The common target function is the mean square error (MSE) criterion, which is expressed as,

$$
\varepsilon(\mathrm{n})=E\left\{e^{2}(n)\right\}=\mathrm{E}\left\{\left[z(\mathrm{n})-\mathrm{W}_{N}(n) \mathrm{Y}_{N}(n)\right]^{2}\right\}
$$

where $E[*]$ is mathematical expectation and $\varepsilon(\mathrm{n})$ is function of $n$. When the state reached steady, the system is convergent. Assumed initial coefficients $\mathrm{w}_{N}(0)$,

$$
W_{N}(\mathrm{n}+1)=W_{N}(n)+\mu \cdot e(n) \cdot Y_{N}(\mathrm{n})
$$

where $\mu$ is iterative steps of the LMS algorithms.

It can be seen that LMS updates the predistortion coefficients through equation (11). Iterations are performed until the difference equal or approximate to zero of equation (5), and then the DPD predistorter direct copy the preditortion coefficients and PA's nonlinear characteristics complementary to achieve linear amplification.

\subsubsection{Proposed LS-LMS}

We all know that the LMS algorithm convergence is sensitive to the properties of step length and the initial value. Especially, the convergence speed is slow when the characteristic initial values spread. On the other hand, if the $\mathrm{N}$ is too big, the LS algorithm compute complexity will enlarge with the $U^{H}$. Hence, we proposed the LS-LMS algorithm which helping to improve the convergence speed of LMS and get over the instability of LS. It adopts the little snapshots $\mathrm{N}(\mathrm{N}<<\mathrm{K}, \mathrm{K}$ is the length of input signal) of LS, computing the weighted vector, and then using the vector as an initial value of SLM algorithm.

The algorithm process is explained as follows. When $0<\mathrm{K} \leq \mathrm{N}$, the LS with small snapshot number $\mathrm{N}$ calculate the weighted vector as

$$
\vec{w}(N)=\left(\mathrm{U}^{H} U\right)^{-1} U^{H} Z
$$

when $\mathrm{K}>\mathrm{N}$, using $\vec{w}(N)$ as an initial value of SLM and then the weighted vector update by LMS algorithm.

$$
W_{N}(\mathrm{k}+1)=W_{N}(\mathrm{k})+\mu \cdot e(\mathrm{k}) \cdot Y_{N}(\mathrm{k})
$$

Through the formula (12) (13) completed weighted vector update by LS- LMS. Since the nonlinear characteristics of PA are variable with temperature fluctuating and device aging. Adaptive algorithm is enabled again LMS algorithm, as long as the difference between $z(n)$ and $z \hat{(n)}$ is not equal or approximate to zero [13].

\section{Proposed New Combined Architecture}

To increase power efficiency and improve linearization, the joint algorithm combing SLM $+\mathrm{CT}$ with DPD is proposed. As illustrated in Fig. 4 and Fig. 5.

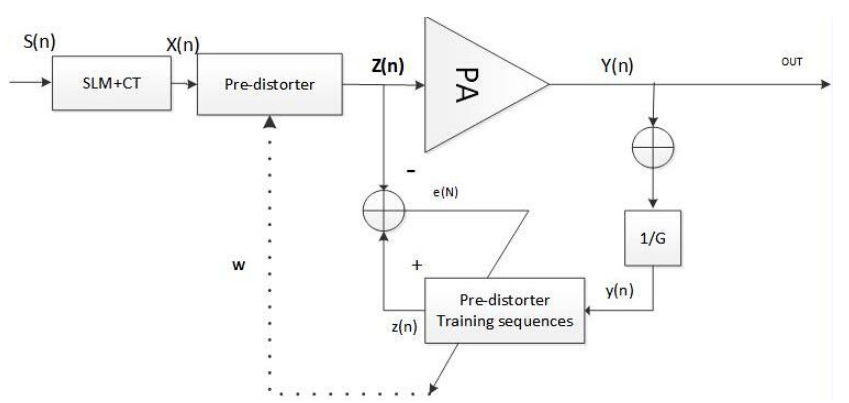

Fig. 4: Systematic scheme of the proposed combined CFR and DPD.

Firstly, the PAPR of input signal $s(n)$ is reduced by SLM. Then, it is reduced further by employing the CT method. While SLM method does not bring distortion to the system but it will miss some peak value of 
the signal. The miss part of peak signals is reducing by CT. The advantage of proposed method is that the CT reduces low peak signal after SLM, which produces littler noise than using the CT individual. In other words, it not only greatly reduced the PAPR but also improved the system's EVM.

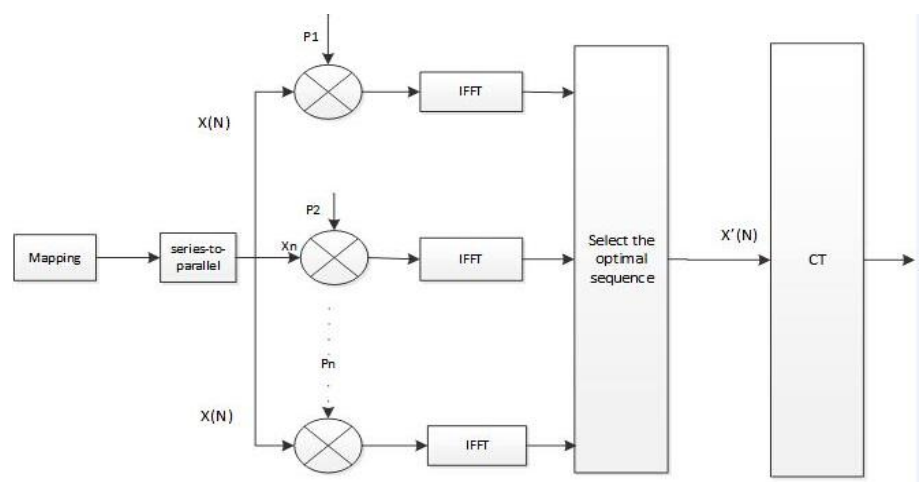

Fig. 5: SLM and CT schematic diagram.

\section{Simulation Results}

In this section, computer simulation with MATLAB software is accomplished. We utilize a LTE signal that is an OFDM signal with bandwidth $20 \mathrm{MHz}, 2048$-point IFFT size, 1200 subcarriers and PAPR $19.03 \mathrm{~dB}$. For the DPD, we select the MP, the polynomial order $\mathrm{K}=5$ and the memory depth $\mathrm{Q}=3$.

PA's nonlinear characteristics can be described as AM/AM and AM/PM. Fig. 6 describe the AM-AM and AM-PM characteristics of curves. Simulation results show that, the AM-AM and AM-PM characteristics without using MP DPD are fuzzy and nonlinear, as show in the blue curves in Fig. 6 After the signal is processed with proposed new combined MPDPD, the characteristics have been greatly corrected, as show in the black curves in Fig. 6.
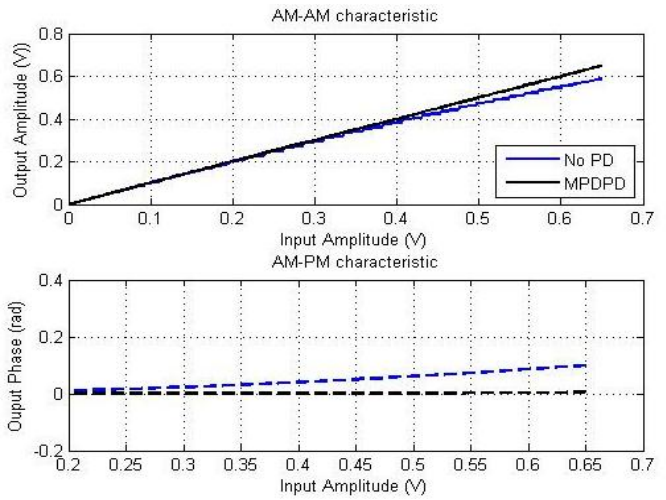

Fig. 6: AM/AM and AM/PM curve.

The LMS error convergence curve and LS-LMS error convergence curve $(\mathrm{N}=50)$ are showed in Fig. 7 , where the abscissa represents the number of iterations and the vertical axis represents the magnitude of error. Simulation results show that the LS-LMS error curve is smooth and convergent fast than LMS algorithm [6].
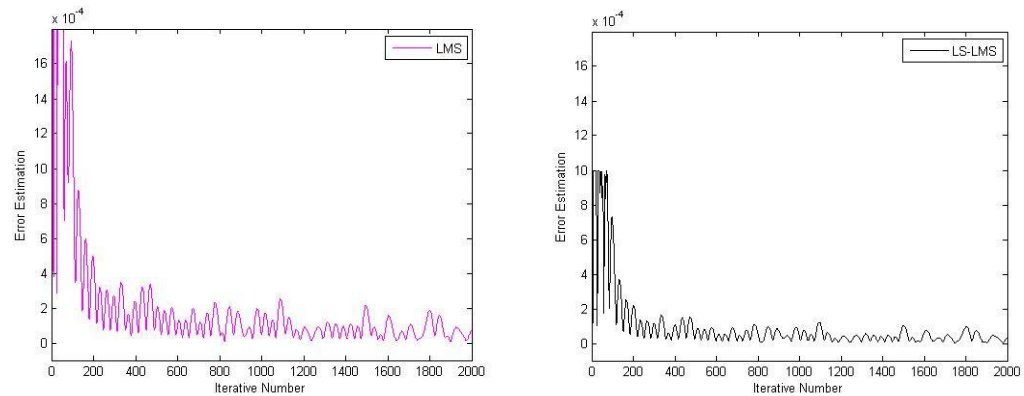

Fig. 7: The error curve.

Simulation and comparison of several schemes included NO DPD, MP-DPD, CT+MP-DPD and SLM+CT+MP-DPD, adjacent channel power ratio (ACPR), Normalized mean square error (NMSE), error 
vector magnitude (EVM) and PAPR are calculated, as shown in table1. When the signal is amplified by PA without DPD, the ACPR, NMSE and EVM is not ideal due to the PA's nonlinearity. Furthermore, the PAPR value reached $19 \mathrm{~dB}$. When the signal is processed by MP-DPD, the EVM, NMSE, ACPR are desirable, but it still has high PAPR. When we process the signal with MP-DPD and CT, the PA input signal's part of PAPR (about $4.3 \mathrm{~dB}$ ) is reduced [6]. Finally, when we joint SLM, CT and MP-DPD, the PAPR is reduced further (about $7 \mathrm{~dB}$ ). At the same time, other performances such as EVM, NMSE, ACPR are improved comparing to the other schemes. Thus, the proposed approach is valuable within a certain range.

Table 1: Performance Comparison of EVM, NMSE, ACPR and PAPR

\begin{tabular}{lllll}
\hline & EVM/\% & NMSE/dB & ACPR/dB & PAPR/dB \\
\hline Without DPD & 10.247 & -9.893 & $-13.090 /-13.091$ & 18.820 \\
MP-DPD & 0.615 & -22.110 & $-18.170 /-18.383$ & 19.086 \\
CT+MP-DPD & 0.432 & -23.643 & $-25.963 /-25.983$ & 14.862 \\
SLM+CT+MP- & 0.375 & -25.266 & $-28.015 /-27.976$ & 11.780 \\
DPD & & & & \\
\hline
\end{tabular}

Finally, the power spectral density of the output signal is showed in the Fig. 8, in which the combination of the adaptive DPD and SLM+CT achieved $11 \mathrm{~dB}$ improvements.

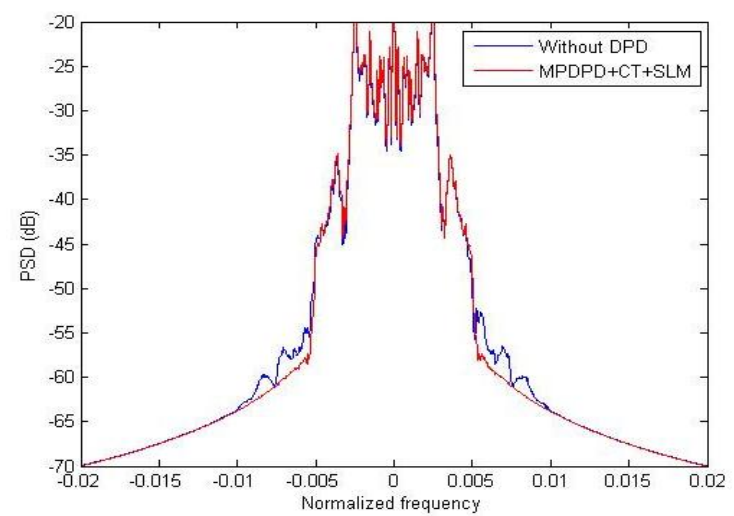

Fig. 8: The PSD of output signal.

\section{Conclusions}

In this paper, a new method of combining SLM+CT and adaptive DPD is proposed. The simulation results show that the MP-DPD with LS-LMS adaptive algorithm effectively lowered the inter-symbol interferences and spectral regrowth caused by the PA's nonlinearity and memory effect. Most importantly, the PAPR is reduced by adding the SLM and CT in the DPD system, and the PA's efficiency has been greatly improved. The proposed method of this paper is simple and feasible. It leads to a significant reduction in implementation cost and system complexity.

\section{References}

[1] Besbes H, Le-Ngoc T, A fast adaptive predistoter for nonlinearly amplified M-QAM signals, IEEE Global Telecommunications Conference, San Francisco,2000.1:108-112.

[2] M.Mahmudul Hasan, A New PAPR Reduction Scheme for OFDM Systems Based on Gamma Correction, Circuits Syst Signal Process (2014) doi.10.1007/s00034-013-9712-2.

[3] R. NeilBraithwaite, ImplementingCrest FactorReduction(CFR) by Offsetting Digital Predistortion(DPD) CoefficientsIEEE Powerwave Technologies, Santa Ana, CA 92705 USA 2012.

[4] Byung Moo Lee and Rui J.P.de Figueiredo, Adaptiv Predistorters For Linearization Of High-Power Amplifiers In OFDM Wireless Communications, Circuits Syst Signal Process(2006). Doi.10.1007/s00034.004-0901-X.

[5] A. Farabegoli,B.sogl and R.weigel ,Advanced Transmitters with combined Crest Factor Reduction and Digital Predistortion Techniques, IEEE RWS 2014. 
[6] Zhinian LUO, Kejian ZHOU, A New Combined Approach of Digital Predistortion and Companding Transform for the nonlinear Distortion Compensation, Journal of Computational Information Systems, 2015vol.11(5):1781-1789.

[7] Byung Moo Lee. Yongnok Ki, An Adaptive Clipping and Filtering Techinque for PAPR Reduction of OFDM Signals. Circuits Syst Signal Process(2013) Doi.10.1007/s0034-012-9512-0.

[8] Lin CHEN, Xulong HU,Improved SLM Techniques for PAPR Reduction in OFDM Systems, Journal of Computational Information Systems,6:13(2010),4427-4423.

[9] Xinchun Wu. Jinxiang Wang .Zhigang Mao, Conjugate Interleaved Partitioning PTS Scheme for PAPR Reduction of OFDM Signals. Circuits Syst Signal Process (2010), Doi 10.1007/s00034-010-9157-9.

[10] Jing Zhang, Songbai He, Shirong Yin, A Memory Polynomial Predistorter for Compensation of Nonlinearity with Memory Effect in WCDMA Transmitters[C], International Conference on Communicational Information Systems, 6:13(2010),4427-4433.

[11]L. Ding, Z. Ma, D. R. Morgan, M. Zierdt, and J. Pastalan, A leastsquares/Newton method for digital predistortion of wideband signals,'IEEE Trans. Commun, vol. 54, no. 5, pp. 833-840, May 2006

[12] Azzedine Zerguine, Qualid Hammo, Behavioral Modeling and Predistortion of nonlinear Power Amplifiers Based on Adaptive Filtering Techniques, IEEE 2014.

[13]Zhibin Zeng, A Crest Factor Reduction Method in Digital Predistortion for Improvement of Power Efficiency, IEEE International Conference on Computer Science and Electronics Engineering, 2012. 